\title{
INTERVENÇÃO FAMILIAR NA ESQUIZOFRENIA: RECORTE DE MODELOS DE ENSAIOS CLÍNICOS
}

\author{
Maria Goretti Andrade Rodrigues ${ }^{\star}$ \\ Letícia Krauss Silva ${ }^{\star \star}$
}

\begin{abstract}
RESUMO
O entendimento do formato da Intervenção Familiar citada pelos ensaios clínicos controlados na condição esquizofrenia foi o foco de nossa investigação aqui relatada. Procedemos a uma busca sistemática na base de dados MedLine e elaboramos a compreensão da fundamentação teórica das práticas descritas para avaliar a heterogeneidade clínica das intervenções que são objeto de estudo dos ensaios que resgatamos. Descrevemos um modelo denominado "pragmático", que apresenta fragmentos de técnicas, sem princípios de base ou técnicas derivadas de teorias; outro modelo cuja ênfase do trabalho é na mudança comportamental; e por fim um modelo cognitivo comportamental, que privilegia a abordagem cognitiva. Fazemos considerações sobre os conceitos de Emoção Expressa (EE) e Sobrecarga Familiar, subjacentes aos modelos comportamental e cognitivo comportamental na Intervenção Familiar para Portadores de Esquizofrenia. Consideramos que esse modelo, que privilegia um maior entendimento do familiar acerca da esquizofrenia, com a devida adequação devido às especificidades culturais nacionais, deve ser investigado para a utilização nos CAPS do Brasil.
\end{abstract}

Palavras-chave: Esquizofrenia. Intervenção Familiar. Sobrecarga Familiar. Avaliação Tecnológica. Centro de Atenção Psicossocial.

\section{FAMILY INTERVENTION IN THE SCHIZOPHRENIA: CUT OUT OF THE MODELS OF CLINICAL TRIALS}

\begin{abstract}
The understanding of the format of the Family Intervention mentioned by the clinical trials controlled in the condition schizophrenia it was the focus of our investigation here told. We proceeded to the one systematic search in MedLine and we elaborated for the understanding of the theoretical foundation of the described practices to

^ Doutoranda em Saúde Pública ENSP / FIOCRUZ. Atuou como Psicóloga do CAPS Ilha da Convivência, em Santo Antônio de Pádua-RJ, no período de 1995 a 2005. Professora do Departamento de Educação Matemática da Universidade Federal Fluminense.

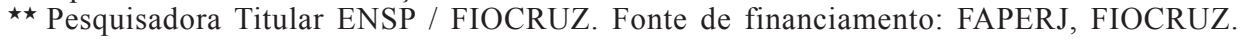
Endereço: Rua Carlos Mâncio de Aquino, 144 - São Luis - Santo Antônio de Pádua - RJ. E-mail: goretti@vm.uff.br
\end{abstract}


evaluate the clinical heterogeneity of the interventions that are object of study of the trials that we rescued. We described a model denominated " pragmatic ", that presents fragments of techniques, without base beginnings or derived techniques of theories; another model whose emphasis of the work is in the change comportamental; and finally a model cognitive comportamental, that privileges the cognitive approach. We make considerations on the concepts of Expressed Emotion (EE) and Family Burden, underlying to the models comportamental and cognitive comportamental in the Family Intervention for Bearers of Schizophrenia.

Keywords: Schizophrenia. Family Intervention. Family Burden. Technological Assessment. Outpatients Care Mental Health.

\section{I - INTRODUÇão}

As barreiras entre o sistema de saúde e as famílias de portadores de esquizofrenia têm sido abordadas na literatura (FALLOON et al, 1985; BARROWCLOUGH; TARRIER, 1997; DIXON, 1999). Os rumos da desinstitucionalização, no Brasil, impõem um maior entendimento das tecnologias consideradas eficazes pela evidência científica, e possivelmente efetivas em nossa realidade, quanto a esse problema.

Apesar dos resultados positivos substanciados por numerosos estudos (DIXON, 1999), a inclusão de familiares na intervenção dirigida à condição esquizofrenia é muitas vezes feita, em nosso país, de forma pouco elaborada, sem apoio nos resultados dos estudos conduzidos, nas últimas décadas, de forma paralela às experiências de desinstitucionalização da atenção aos portadores daquela condição.

Frente à necessidade de construir subsídios para o planejamento da intervenção familiar (IF) nos Centros de Atenção Psicossocial (CAPS), o presente estudo utilizou princípios da avaliação tecnológica. Dado o custo crescente das intervenções em saúde, considera-se atualmente que as tomadas de decisão relativas à incorporação e ao financiamento de tecnologias/procedimentos de assistência à saúde, assim como a produção, difusão e implementação de normas de conduta clinica, devem ser subsidiadas por avaliações tecnológicas que sintetizem as evidências científicas existentes sobre os efeitos das tecnologias de atenção à saúde. Todavia, o modo pelo qual as tecnologias de atenção à saúde têm sido introduzidas/ transferidas para países em desenvolvimento tem sido permeado por numerosas falhas. Os poucos estudos existentes mostram que o processo de incorporação de tecnologias na área de saúde em paises em desenvolvimento tem conduzido à baixa efetividade e baixa eficiência dos recursos empregados na assistência (KRAUSS-SILVA, 2004).

A principal fonte de análise da evidência citada pelo documento programático do governo americano (U.S. DEPARTMENT OF HEALTH AND HUMAN SERVICES, 1999) são os achados dos estudos conduzidos pelo Grupo de Pesqui- 
sa de Resultados relativos a portadores de Esquizofrenia, formado em 1992, pelo governo dos EUA (LEHMAN et al., 1998), o qual teve como objetivos desenvolver e disseminar recomendações para o tratamento da esquizofrenia baseadas em evidências científicas.

Segundo as diretrizes do referido grupo, uma intervenção psicossocial familiar deveria ser oferecida a pacientes que têm contato contínuo com seus familiares como também a cuidadores que não fossem da família. Essa diretriz estaria apoiada em achados de ensaios clínicos controlados que teriam repetidamente demonstrado que a intervenção familiar, associada à farmacoterapia, reduziria índices de recaída de uma média anual de 40 a 53\% para uma média de 2 a 23\% (DIXON; LEHMAN, 1995).

Outra diretriz produzida pelo Grupo (LEHMAN et al., 1998) foi a de que terapias de família baseadas na premissa de que disfunção familiar seria a etiologia do transtorno esquizofrênico no paciente não deveriam ser usadas, uma vez que os estudos disponíveis, embora limitados, não teriam dado apoio à hipótese de relação causal entre disfunção familiar e etiologia da esquizofrenia, não havendo evidência de efetividade das terapias desenhadas com tal premissa (DIXON; LEHMAN, 1995).

A análise das características dos procedimentos de intervenção familiar (IF) investigados pelos estudos clínicos pode contribuir, enquanto etapa do processo de revisão sistemática de efeitos da IF, para definir modelo(s) cuja transferência tenha maior probabilidade de sucesso em nossos CAPS.

\section{II - OвJETIVo}

Analisar a evolução histórica dos procedimentos de intervenção familiar (IF) investigados para a condição esquizofrenia, classificando-os, particularmente, quanto a técnicas envolvidas, a partir de referenciais teórico-metodológicos, e a outras características relevantes, como tipo de paciente/familiar, duração da IF e desfechos analisados.

\section{III - Metodologia}

Na busca realizada no MedLine com as palavras-chave family therapy AND schizophrenia, e os limites Title/Abstract, Clinical Trial, Human, Publication Date from 1970 to 2005, encontramos 21 ensaios randomizados.

A análise das propostas de IF dos ensaios foi baseada em teorias, métodos e técnicas comportamentais e cognitivo-comportamentais previamente analisados (BANDURA, 1977; BECK, 1979; CABALLO, 1996; DOBSON; DOZOIS, 2001). Com base nesses referenciais, foi então considerado "comportamental" o ensaio de IF que apresentasse técnicas baseadas na teoria do condicionamento clássico, na teoria do condicionamento operante ou, e especialmente, na teoria da Aprendizagem Social, privilegiando dentro desse último grupo os procedimentos do treinamento de habilidades sociais. 
Consideramos "cognitivo-comportamental" o ensaio cuja abordagem na IF: 1) apresentasse as seguintes proposições fundamentais (DOBSON; DOZOIS, 2001): a) a atividade cognitiva afeta o comportamento (modelo mediacional), b) a atividade cognitiva pode ser monitorada e alterada, e c) a mudança do comportamento desejada pode ser alcançada através da mudança cognitiva; 2) aplicasse as terapias baseadas no método cognitivo-comportamental: terapias de reestruturação cognitiva, terapias de habilidades para lidar com dificuldades e terapias de resolução de problemas.

BECK (1979) esclarece que o ponto focal dessas terapias difere sobretudo porque o terapeuta cognitivo-comportamental direciona sua técnica para modificar o "conteúdo ideacional" envolvido no sintoma, através da "mediação cognitiva".

\section{IV - Resultados: EvoluÇão históRICA E TIPOLOGIA}

Foram delimitados 4 subgrupos de ensaios de acordo com o método/técnica utilizado: linha "pragmática", linha comportamental, linha cognitivocomportamental e não definidos.

\section{LINHA "PRAGMÁTICA":}

O grupo de ensaios denominados "pragmáticos" por alguns autores é baseado no modelo estresse-diátese da esquizofrenia e apresenta características comuns quanto aos objetivos e semelhanças de conteúdos/extensão da intervenção proposta, e quanto ao fato de não constituírem uma técnica, derivada de base teórico-metodológica definida. Nesse grupo, a IF teve como principal finalidade diminuir o estresse do paciente, visando evitar a recaída deste.

Os precursores dessa linha de ensaios (GOLDSTEIN et al., 1978), propõem 6 sessões com a família, orientadas para o entendimento da doença e da relação entre estresse e surto (inclusive recaída), identificação de situações de estresse e planejamento no sentido de evitar ou minimizar tais situações. Não explicita se a IF é direcionada para grupos de famílias ou para famílias únicas. As técnicas e atividades envolvidas não são explicitadas pelo ensaio.

Os ensaios posteriores da linha "pragmática" (LEFF et al., 1982, 1985; GLICK et al., 1985; HAAS et al., 1988; HOGARTY et al., 1986, 1991; LEFF et al., 1989, 1990; MCFARLANE et al., 1995; TOMARAS et al., 2000) testam uma IF ainda com o foco apenas no paciente. A família é vista como co-terapeuta e como fator prognóstico de recaída pela variável "emoção expressa" (EE) dos familiares em relação ao paciente, medida pelas respostas emocionais "comentários críticos", "hostilidade" e "superenvolvimento emocional" (BROWN; BIRLEY; WING, 1972; VAUGHN; LEFF, 1976).

Antes de examinar as IFs propostas pelos ensaios da linha pragmática, registramos sucintamente o trabalho de Anderson et al. (1980), que é citado por todos os ensaios dessa linha como modelo para alguma etapa das IFs realizadas. Ele teve por base estudos que identificaram um "déficit psicológico central" em certos portadores de esquizofrenia, que apareceria como uma disfunção do pro- 
cesso de atenção que seria afetado adversamente por extremos de estímulos, manifestada pela intensidade das respostas a tais estímulos. Os comportamentos resultantes dessa disfunção corresponderiam a dificuldades perceptivas e cognitivas que seriam compatíveis com os principais sinais da esquizofrenia. Tais déficits no processamento de estímulos poderiam ser manifestados por estímulos dos ambientes natural e terapêutico do paciente. Ainda segundo outros estudos, algumas famílias teriam padrões de interação - por exemplo: falta de clareza, de reconhecimento, comportamentos comunicativos vagos ou dissociados, ou ainda "comportamentos críticos" ou com "super-envolvimento", estes últimos também chamados de "emoção expressa" - que poderiam exacerbar o déficit sugerido e propiciariam a recaída do paciente. Ou seja, haveria um ciclo vicioso de hiperestimulação, distração, desatenção e doença no paciente e frustração e desesperança crescentes na família. A proposta dos autores é propiciar um ambiente mais benigno para o paciente que seria um ambiente modificado do ponto de vista de estímulos.

Os objetivos da IF proposta por Anderson et al. (1980) seriam melhorar a comunicação da família e diminuir sua emoção expressa (EE) (nos casos de familiares de alta EE), visando a prevenção da recaída. Os autores não explicitam técnicas, mas descrevem as atividades sucessivas da IF:

Fase I - Conexão com a Família: reuniões para estabelecer conexão com a família. Começa logo após a admissão hospitalar, ou tão cedo quanto possível, envolvendo pelo menos 2 sessões por semana durante a fase aguda/hospitalização;

Fase II: ensino de habilidades de sobrevivência para lidar com a esquizofrenia ("Survival Skills Workshop") através de uma oficina de um dia inteiro com múltiplas famílias (4 a 5 famílias), com objetivos de: 1) repasse de informações sobre esquizofrenia (fenomenologia, instalação, tratamento, curso e resultado) em linguagem clara, 2) informações sobre formas de lidar com a esquizofrenia, 3) modificação (melhora) da comunicação e 4) atenção do familiar a si próprio, para promover a re-socialização (de-isolation) da família, dessensibilização e normalização da questão do transtorno mental na família. O paciente em surto não participaria da Oficina. A Oficina também propiciaria a exposição das formas de lidar com o transtorno mental pelas diferentes famílias, o que possibilitaria, segundo os autores, a comparação entre essas formas de lidar dos familiares e uma aprendizagem sobre tal enfrentamento da situação. O trabalho de grupo e a secção de atenção a si próprio propiciariam ainda a formação de rede (artificial) de suporte social.

Fase III: trabalho direcionado a família única com a presença do paciente (sessões semanais/bi-semanais durante, pelo menos, seis meses), onde a maneira de usar habilidades de sobrevivência anteriormente trabalhada em grupo é aplicada às situações e preocupações específicas da família/paciente (comportamentos do paciente que são problema para a família e vice-versa), relacionadas principalmente a dois temas: o reforço de limites intrafamiliares (aumento do espaço psicológico do paciente) e o reassumir gradual das responsabilidades por parte do paciente; 
Fase IV: por fim, a opção do familiar pelo engajamento em "terapia familiar tradicional", com a exploração de estratégias de interação familiar, com foco nos outros problemas familiares.

Notamos, nos quatro itens da fase II e na fase III, rudimentos da técnica comportamental de Treinamento de Habilidades Sociais. A proposta de Anderson et al. e, particularmente, a etapa relativa à comparação de formas de lidar não constituem técnica/procedimento comportamental de treinamento de habilidades sociais/modelagem, porque o procedimento de modelagem aparece dissociado de outros procedimentos comportamentais (como reforço e ensaio comportamental) e é trabalhado aqui de forma assistemática. Embora inclua repasse de informação, não percebemos, nessa proposta de IF, indícios de enfoque na mediação cognitiva, portanto também não a caracterizamos como procedimento cognitivocomportamental.

O ensaios "pragmático" de Glick et al. (1985) / Haas et al. (1988) testa IF que, segundo os autores, foi baseada em sua experiência clínica e nos trabalhos de Goldstein et al. (1981 apud GLICK et al., 1985) e de Anderson et al. (1980). Todavia, as atividades são muito semelhantes às propostas por Goldstein et al. (1978), sendo acrescentado àquela a especificação da elucidação de seqüências de interação familiar que produzem estresse no paciente.

Os demais ensaios que classificamos como "pragmáticos" (LEFF et al., 1982, 1985 (placebo-controlado); LEFF et al., 1989, 1990 (comparativo); Hogarty et al., 1986, 1991; MCFARLANE et al., 1995 (comparativo); TOMARAS et al., 2000) tiveram como objetivo principal a mudança do nível da variável emoção expressa (EE) dos familiares (de alta para baixa), visando diminuir o estresse do contexto do paciente, para evitar recaída, o que incorpora em boa parte os objetivos da IF proposta por Anderson et al. (1980).

Embora refira ter sido baseado no modelo de Anderson et al. (1980), o ensaio de Leff et al. $(1982,1985)$ não inicia ou especifica uma etapa de conexão com a família do paciente; por outro lado, o repasse de informações sobre a doença constitui uma etapa específica e inicial, com material instrutivo mais elaborado e trabalhado em 4 aulas. Também o contato entre famílias, para relato/exposição de formas diferentes de lidar com o paciente, teve duração mais longa, espaçado em sessões relativamente curtas, mas foi complementado, como em Anderson et al. (1980), por sessões unifamiliares onde esse trabalho foi especificado e ampliado.

O ensaio mais tardio de Leff et al. $(1989,1990)$ e o de Mcfarlane et al. (1995) são comparativos de IFs para grupos multi (6 famílias) e uni familiares, ambas as alternativas constituídas de alguns elementos da proposta de Anderson et al. (1980).

No ensaio de Hogarty et al. (1986, 1991), identificamos elementos rudimentares de procedimentos do método comportamental, como reforço (baseado no condicionamento operante), treinamento de habilidades de comunicação e resolução de problemas (procedimentos baseados na teoria de aprendizagem soci- 
al). Esses procedimentos não aparecem, todavia, como o centro do trabalho de Hogarty et al. $(1986,1991)$ mas associados a um trabalho educativo, como nas fases II e III do modelo de Anderson et al. (1980).

O ensaio de Mcfarlane et al. (1995) apresenta como componentes sessões de engajamento, tal qual a primeira fase, de conexão, no modelo de Anderson et al. (1980), seguidas pelo Survival Skills Workshop, ou seja, a fase II do mesmo modelo. Enquanto componente, é ainda utilizado o procedimento de resolução de problemas, essencialmente cognitivo, mas referido ao manual de Falloon et al. (1984) (que utiliza técnica comportamental, conforme veremos adiante), nos dois grupos comparados, com a diferença de que no grupo unifamiliar de IF o terapeuta tem mais envolvimento no trabalho e acompanha a resolução de problemas mais específicos de cada família. Os autores não mencionam, todavia, a utilização de outros procedimentos nem a articulação dos componentes apresentados e, por isso, nenhuma das alternativas comparadas deveria ser classificada como técnica comportamental.

Finalmente, no grupo dos ensaios classificados como "pragmáticos", o de Tomaras et al. (2000) menciona a utilização de um "modelo psicoeducacional" que estaria explicitado no guidelines of psychoeducational model, de Anderson et al. (1986 apud TOMARAS et al., 2000). Do que é descrito, a IF constou de 13 sessões de grupo multifamiliar ( 6 a 8 familiares-chave) que incorporaram elementos dos demais ensaios "pragmáticos", sendo bastante semelhante aos ensaios de Leff et al. $(1982,1985)$. Os sujeitos foram portadores de esquizofrenia cronificados, enquanto que a maioria dos ensaios pragmáticos teve percentual importante de pacientes em estágios iniciais.

Quanto a métodos de tratamento, o que podemos apreender dos ensaios "pragmáticos" é que as IFs testadas constituem fragmentos de técnicas, ou seja, pedaços de procedimentos utilizados sem a lógica e a articulação previstas.

\section{LINHA COMPORTAMENTAL:}

No início dos anos 80, Falloon e outros $(1982,1984,1985)$, que mantêm o paradigma estresse-diátese, aparecem como divisor de águas ao ampliar o foco da IF além do paciente, para a família e seu bem-estar. Embora também percebam o potencial reabilitativo da família no tratamento do portador de esquizofrenia, vêem aquela como altamente atingida pela condição, sobrecarregada em vários aspectos, sendo que esse mal-estar necessitaria ser objeto da atenção à condição, no sentido de evitá-lo, minimizá-lo. O desfecho (resultado) "sobrecarga familiar" aparece, pela $1^{\mathrm{a}}$ vez, nesse ensaio.

Os objetivos do ensaio de Falloon et al. $(1982,1985)$ são ensinar aos pacientes e familiares métodos para lidar com eventos estressantes e a mudança de atitudes de familiares-chave em direção a maior suporte, menos críticas destrutivas, e menos envolvimento emocional (EE) para com o portador de esquizofrenia. A IF testada enfatiza a mudança comportamental utilizando, a nosso ver, a técnica do "treinamento em habilidades sociais" (THS), que pode ser definido como um 
enfoque terapêutico geral dirigido a incrementar a competência da atuação em situações críticas da vida ou como uma tentativa direta e sistemática de ensinar estratégias e habilidades interpessoais aos indivíduos, com a intenção de melhorar sua competência interpessoal e individual em situações sociais específicas (CABALLO, 1996). O THS se adere a um enfoque comportamental de aquisição de resposta - isto é, concentra-se na aprendizagem de um novo repertório de respostas, abrangendo quatro elementos de forma estruturada:

1.Treinamento de habilidades: ensino de comportamentos específicos, que são praticados e integrados ao repertório comportamental do sujeito. Dado que a aquisição dessas habilidades depende de um conjunto de procedimentos derivados, principalmente, da teoria da aprendizagem social, o THS inclui muitos desses procedimentos em sua aplicação, como instruções, modelagem, ensaio comportamental, feedback e reforço. 2. Redução da ansiedade em situações sociais problemáticas, a ser conseguida de forma indireta, ou seja, ocorrendo o novo comportamento mais adaptativo que, supostamente, é incompatível com a resposta de ansiedade. Se o nível de ansiedade é muito elevado, podese empregar diretamente uma técnica de relaxamento ou a dessensibilização sistemática. 3. Reestruturação cognitiva, na qual se pretende modificar valores, crenças, cognições e/ou atitudes do sujeito. A reestruturação cognitiva ocorre também de forma indireta, isto é, a aquisição de novos comportamentos modifica, a longo prazo, as cognições do sujeito. Aqui não se trata do subtipo de terapia cognitiva típica do método cognitivo-comportamental, mas de um procedimento cognitivo auxiliar, com menor grau de complexidade. 4. Treinamento em solução de problemas, que ensina o sujeito (familiar) a perceber corretamente os "valores" de todos os parâmetros situacionais relevantes, a processar os "valores" destes parâmetros para gerar respostas potenciais, a selecionar uma dessas respostas e a enviá-la de modo que maximize a probabilidade de alcançar o objetivo que impulsionou a comunicação interpessoal. Embora o treinamento em solução de problemas, procedimento cognitivo, não ocorra de forma sistemática nos programas de THS, geralmente se encontra presente neles, de maneira implícita, sendo articulado, nessa técnica, como procedimento complementar e com grau de complexidade relativamente baixo.

A IF testada por Falloon e outros $(1982,1985)$ se constitui, conforme os autores, de procedimentos comportamentais da técnica THS: seleção (inclusão no ensaio) de familiares considerados de alta EE ou de famílias com elevação do nível de tensão (etapa do THS de identificação da classe de comportamento-problema), educação (paciente e família) sobre a natureza, curso e tratamento da esquizofrenia (etapa do THS de reestruturação cognitiva), ensaio comportamental, modelagem, reforço social e avaliação (correspondentes à etapa do ensaio comportamental do THS).

Os autores relatam que tais procedimentos foram usados para aumentar habilidades de expressão de sentimentos positivos e negativos, de escuta reflexiva 
(olhar, expressão facial, gestos e postura), de demanda para mudança de comportamento (orientação), e de reciprocidade de conversação (distância/contato físico, volume da voz, entonação, fluência, tempo de fala, e conteúdo), ou seja, para modificar elementos "moleculares" do comportamento, importantes no âmbito das habilidades sociais, segundo Caballo (1996).

O procedimento resolução de problemas, cognitivo, utilizado por Falloon e outros $(1982,1985)$, pode ser considerado procedimento auxiliar da técnica comportamental visto que se dá em paralelo e de forma complementar com outros procedimentos do THS, ou seja, seguindo o modelo de articulação de procedimento cognitivo na terapia comportamental e possuindo um grau de complexidade menor que o da técnica cognitiva. Os pacientes/familiares foram incluídos nesse ensaio após a ocorrência de um surto, mas só depois da estabilização de sintomas e funcionamento social, e sob medicação adequada. Não há informação se a intervenção foi no primeiro surto, apenas sobre a média de idade (26anos).

Três outros ensaios referem que tomaram como base o trabalho de Falloon et al.. Os objetivos da IF testada por Mueser et al. (2001) foram o ensino de habilidades para o gerenciamento da esquizofrenia e para diminuir estresse intra-familiar. Os autores referem o uso de procedimentos de treinamento de habilidades de comunicação e de resolução de problemas para a obtenção de metas colaborativamente, em atividades uni e multifamiliares. Apesar de referirem o manual de Falloon et al., e de terem sido treinados pelo próprio Falloon e seus colaboradores, esse ensaio difere na identificação do comportamento-problema pois não seleciona famílias de alta $\mathrm{EE}$, mas famílias que tivessem um mínimo de 4 horas de contato regular face-a-face por semana. Os pacientes (familiares) incluídos no ensaio de Mueser et al. foram recrutados durante exacerbação de sintomas e a IF teve início após estabilização, via medicamentos, dos sintomas.

O ensaio comparativo de Montero et al. (2001; 2005), também referido a Falloon et al. (1984), não informou sobre a cronicidade dos pacientes; a IF foi iniciada após a estabilização clínica dos pacientes, com remissão de sintomas psicóticos positivos e capazes de acompanhar as sessões e processar informação em um grau razoável. Apresenta IF com procedimentos comportamentais como treinamento em habilidades de comunicação e aprendizagem e prática de técnicas de resolução de problemas, também tendo como referência o manual de Falloon et al. (1985). A eficácia da IF comportamental para grupo de múltiplas famílias é comparada com grupo de familiares, que seguiu o modelo de Leff et al. (1990), que classificamos como "pragmático".

Finalmente, o trabalho de Xiong et al. (1994), realizado na China, teve como objetivos aumentar o funcionamento ocupacional dos pacientes, inclusive, a prevenção da recaída, e a colaboração familiar com a medicação dos pacientes para diminuir a sua sobrecarga. Informam que mais de $90 \%$ dos pacientes incluídos no estudo apresentava o transtorno mental há mais de um ano. A IF constituiu-se de três fases, sendo a $1^{\mathrm{a}}$ realizada durante a hospitalização, quando foram oferecidas informações aos familiares sobre esquizofrenia e foi trabalhada uma estratégia efetiva de gerenciamento de medicação e estabelecida uma relação de empatia. 
A $2^{\mathrm{a}}$ fase objetivou melhorar o "funcionamento social" do paciente e desenvolver uma efetiva estratégia de convivência da família. Consistiu de sessões unifamiliares com a presença do paciente e de membros familiares co-residentes, combinadas a sessões multifamiliares, sessões individuais para pacientes e membros familiares e a visitas domiciliares. Os autores referem o uso de procedimentos comportamentais, como treinamento comportamental e resolução de problemas associados ao aconselhamento. A $3^{\mathrm{a}}$ fase, de manutenção, objetivou reduzir a medicação até a dosagem efetiva mais baixa com sessões a cada dois ou três meses para supervisão da medicação e acompanhamento do monitoramento de sintomas. Os procedimentos referidos na $2^{\mathrm{a}}$ fase desse ensaio indicam tratar-se de uma intervenção de base comportamental, embora os autores não apresentem detalhes sobre a técnica utilizada.

\section{LINHA COGNITIVO-COMPORTAMENTAL:}

O ensaio de Tarrier et al. (1988) compara dois tipos de IF, a comportamental participativa e a comportamental simbólica. Ambas são apresentadas como didáticas, com o objetivo primeiro de ensinar familiares habilidades para administrar a esquizofrenia, identificando componentes de alta EE nos familiares e eliminandoos através da mudança no comportamento do familiar ou do paciente, comportamento esse que detonaria a resposta negativa. O segundo objetivo seria encorajar um aumento no nível de funcionamento do paciente, através da identificação sistemática de necessidades e planejamento de metas para atingir essas necessidades. Os pacientes e familiares foram selecionados após admissão hospitalar na fase aguda do transtorno, $30 \%$ dos pacientes haviam sofrido o $1^{\circ}$ episódio. A duração média do transtorno à época da inclusão foi de 6,3 anos.

As etapas de inclusão no ensaio e de educação foram comuns às duas intervenções comparadas: inclusão no ensaio de familiares considerados de alta EE (identificação do comportamento-problema, que também pode ser considerada etapa da terapia cognitivo-comportamental), educação para o paciente e a família com informação ampla e individualizada sobre esquizofrenia e como administrála no ambiente doméstico (procedimento de reestruturação cognitiva). A IF participativa incluiu ainda o programa de gerenciamento de estresse, realizado em 3 sessões, com o objetivo de ensinar familiares a monitorar fontes de estresse e suas reações para com as mesmas e então aprender estratégias mais apropriadas de lidar com as dificuldades, inclusive a recaída do paciente (terapia cognitivocomportamental "de habilidades para lidar com dificuldades" -THLD), que apresenta a mediação cognitiva como centro do trabalho realizado. Finalmente, ambas as IFs testadas utilizaram o "programa de estabelecimento de metas", em 8 sessões, no qual pacientes e familiares são ensinados a identificar áreas de mudança ou necessidade, e estabelecer procedimentos para obter as metas dentro do que os autores denominam de abordagem construtiva (GOLDIAMOND, 1974 apud TARRIER et al., 1988) (correspondente à "terapia de resolução de problemas", método cognitivo-comportamental).

A diferença entre as duas IFs do estudo acima está em como essas habilidades são ensinadas: se através de representação simbólica, tal como instrução e 
discussão, ou através de um método participativo que requer procedimentos complementares comportamentais, com maior participação através de troca de papéis, auto-registro, ensaio comportamental, guia prático etc, e grande envolvimento por parte de familiares na aprendizagem de novos comportamentos. Por exemplo, um familiar seria aconselhado a relaxar mais quando estivesse sob estresse e seria instruído verbalmente em como fazer isso (IF comportamental simbólica), ou seria ensinado ativamente a relaxar sendo fornecido a ele exercícios gravados para praticar, e solicitado a monitorar e registrar o sucesso da prática e sua implementação em situações estressantes (IF participativa).

A IF participativa foi desenvolvida em um nível de complexidade que incluiu procedimentos complementares comportamentais, como troca de papéis, ensaio comportamental, guia prático e auto-monitoramento. A IF simbólica foi operada num nível puramente simbólico.

Portanto, embora a ênfase de tais intervenções seja na mudança comportamental, a mediação cognitiva é a principal estratégia das IFs do ensaio de Tarrier et al. (1988) para alcançar tal mudança, o que caracteriza as IFs do ensaio como cognitivo-comportamentais.

A IF testada no ensaio de Sellwood et al. (2001) não selecionou famílias com base na variável EE e a sobrecarga familiar (BARROWCLOUGH et al., 1998) foi um dos objetivos da IF. O foco, conteúdo e quantidade de sessões da intervenção são determinados através de avaliação sistemática das necessidades identificadas nos cuidadores (familiares) através da aplicação de um instrumento, a versão para familiares da Cardinal Needs Schedule (RCNS). Para cada área de problemas potenciais para cuidadores, foram utilizadas diferentes abordagens de intervenções psicossociais: técnicas de resolução de problemas (FALLOON et al. 1984), intervenções comportamentais-cognitivas com familiares (BARROWCLOUGH; TARRIER, 1997), e intervenções comportamentaiscognitivas individuais (HADDOCK et al., 1998 apud BARROWCLOUGH et al., 1999). Para algumas necessidades, apenas um procedimento (sub-técnica) foi considerado relevante, por exemplo, a necessidade de informação sobre a doença requereu uma intervenção educacional e a prevenção de recaída requereu uma intervenção de monitoramento dos sintomas prodrômicos.

Foram realizadas entre 10 e 20 sessões de IF, dependendo da natureza e número das necessidades identificadas dentro do período de 12 semanas de tratamento. A duração do transtorno à admissão no estudo deveria ser de pelo menos dois anos. O momento da intervenção não é explicitado.

\section{LINHA NÃO DEFINIDA/ ENSAIOS SINGULARES:}

Finalmente, na busca realizada, encontramos alguns ensaios de difícil classificação quanto a método e técnica ou com propostas de aplicação singulares, ou não explicitadas. O ensaio de Mönking (1994) testa uma IF com o conteúdo de grupo de auto-ajuda para familiares de portadores de esquizofrenia. O de Chien e Chan (2004) compreende atividades de grupo de apoio mútuo, não esclarecidas, e de grupo de psicoeducação (referência a MCFARLANE et al., 1995, abordado 
anteriormente). O ensaio de Szmukler et al. (1996) apresenta como conteúdo da IF um "aconselhamento familiar", com dois componentes principais: educativo e ajuda a cuidadores (familiares) no desenvolvimento de "estratégias para lidar com as dificuldades frente aos problemas do paciente, identificadas nas duas sessões iniciais", que poderíamos caracterizar, pela nomenclatura, como "terapia de habilidades para lidar com dificuldades". Porém, não há menção da utilização de técnicas aplicadas em graus diferenciados de dificuldade, o que facilitaria o enfrentamento de situação de alto nível de estresse e caracterizaria a citada terapia (DOBSON; DOZOIS, 2001).

A IF de Vaughan et al. (1992) objetivou melhorar a resolução de problemas e as habilidades de comunicação entre os familiares. Estes foram treinados a definir um problema, gerar soluções, avaliar conseqüências e fazer acordo sobre uma estratégia. Foram dados exercícios para casa e os resultados foram revistos nos encontros subseqüentes. Todavia, os autores não relatam a utilização de outros procedimentos comportamentais que contribuíssem para conformar alguma das técnicas comportamentais conhecidas. Na discussão, explicitam que não houve uma orientação exclusivamente comportamental, sem esclarecer, no entanto, qual outra orientação teria sido utilizada. Também não o caracterizamos como cognitivo-comportamental porque não há detalhamento, no ensaio, da complexidade da "resolução de problemas" que essa técnica cognitiva compreende. A abordagem parece mais uma "bricolage" de procedimentos antes que uma técnica propriamente dita.

Outros ensaios realizados na China (ZHANG et al., 1994; RAN et al., 2003) desenvolveram modelos que consideraram apropriados para aquele país, considerando aspectos peculiares de sua cultura, incluindo educação familiar e sessões de aconselhamento, mas não detalham procedimentos/técnicas utilizadas.

A IF do ensaio de Merinder et al. (1999) teria sido baseada num método "didático interativo" e focalizou os seguintes tópicos: educação sobre a condição esquizofrenia, incluindo diagnóstico, prognóstico, sintomas, medicação (benefícios e efeitos colaterais); tratamento psicossocial; estresse e primeiros sinais de recaída, plano de emergência; possibilidades de atuação do paciente e da família; leis e regulamentos. Não há outras informações acerca da IF em pauta. Por último, o ensaio de Shimodera et al. (2000) utilizou sessões de terapia familiar dirigidas à família única, cujo conteúdo não é explicitado, não possibilitando também a apreensão de elementos para classificação da intervenção quanto a técnicas utilizadas.

\section{V - ConCLusões}

A análise das propostas de IF testadas por ensaios clínicos permitiu perceber mudanças ao longo do tempo nos objetivos, conteúdo/extensão e métodos/ técnicas da Intervenção Familiar, bem como no tipo de pacientes/famílias incluídos nos estudos e nos desfechos estudados. Essa análise foi elaborada com base nas teorias, métodos e técnicas pertinentes, comportamentais e cognitivo- 
comportamentais (BANDURA, 1977; BECK, 1979; CABALLO, 1996; DOBSON; DOZOIS, 2001).

O exame dos tipos/modelos de IF investigados por esses ensaios clínicos, a partir daqueles referenciais, possibilitou a elaboração de uma tipologia das intervenções desenvolvidas e contribuiu para um maior entendimento da evolução histórica da IF e das técnicas testadas consideradas mais eficazes.

As técnicas são procedimentos desenhados e articulados conforme teorias e métodos, e no grupo de ensaios pragmáticos não localizamos princípios e técnicas correspondentes a teorias e métodos, particularmente a métodos de natureza comportamental ou cognitivo-comportamental. Não foi possível classificar alguns dos 21 ensaios do ponto de vista de método/técnica utilizada. $\mathrm{O}$ ensaio de Xiong et al, que classificamos como comportamental, não detalha da forma desejável a técnica utilizada; também os ensaios de Hogarty et al e o de Macfarlane et al, que classificamos como "pragmáticos", não são típicos daquela linha.

O conjunto de ensaios evolui de uma linha com rudimentos de procedimentos/técnicas comportamentais, para ensaios de técnicas com características comportamentais e, finalmente, para ensaios cognitivo-comportamentais, embora existam ensaios recentes que não focalizam a mediação cognitiva, característica do método cognitivo-comportamental, nem técnicas comportamentais propriamente ditas.

O constructo Emoção Expressa (EE), variável identificada pelo número de comentários críticos, grau de hostilidade e superenvolvimento para com o portador de esquizofrenia (VAUGHN; LEFF, 1976), foi utilizado na seleção de pacientes e na medida de desfecho/resultado da IF na família nos ensaios caracterizados como pragmáticos mas tem sido abandonado mais recentemente. No início da década de 80, quando o ensaio de Falloon et al. (1982) foi iniciado, havia indícios de relação de causalidade entre emoção expressa (EE) dos familiares e recaída em portadores de esquizofrenia. Num momento posterior, segundo Falloon; Pederson (1985), a maior justificativa para uma intervenção familiar era que a unidade familiar atuava como ponto de apoio na reabilitação comunitária de portadores de transtorno mental. A metanálise de Butzlaff; Hooley (1998), com base em estudos mais recentes sobre EE, indica que dever-se-ia elaborar um novo entendimento sobre o fenômeno (EE), diferente daquele de fator prognóstico de recaída, já que a EE ocorreria por mecanismos diversos dos sugeridos a partir das observações dos primeiros estudiosos (BROWN et al., 1972; VAUGHN; LEFF, 1976). A diminuição da sobrecarga familiar (SCHENE et al, 1994, 1998; BARROWCLOUGH et al., 1998) tende a ser o objetivo e desfecho da IF na família nos ensaios mais recentes, trazendo o familiar para a cena não mais como coadjuvante a ser trabalhado em suas mudanças de atitude com relação ao paciente, mas como ator principal de sua própria saúde.

Os ensaios de base comportamental privilegiaram técnicas de treinamento em comunicação e resolução de problemas e buscam a transformação das atitudes familiares, aderindo a um enfoque comportamental de aquisição de resposta, isto 
é, concentram-se na aprendizagem de um novo repertório de respostas, sofrendo grande influência da teoria da Aprendizagem Social (BANDURA, 1977).

A abordagem da família com técnicas cognitivo-comportamentais, mais recentes, focalizaram as necessidades dos familiares e seus sentimentos para com o portador de esquizofrenia. Foi considerado cognitivo-comportamental o ensaio cuja abordagem na IF considerou que a atividade cognitiva afeta o comportamento (modelo mediacional), pode ser monitorada e alterada, e que a mudança do comportamento desejada pode ser alcançada através da mudança cognitiva, tendo por base o método de Beck (1979).

Consideramos que devam ser analisados os resultados obtidos pelos ensaios de boa qualidade de modelos de IF de base cognitivo-comportamental, que privilegiam um maior entendimento do familiar acerca da esquizofrenia, com a devida adequação devido às especificidades nacionais, com vistas a sua eventual utilização nos CAPS do país.

\section{REFERÊNCIAS}

ANDERSON, C. M.; HOGARTY, G. E.; REISS, D.J. Family treatment of adult schizophrenic patients: a psycho-educational approach. Schizophrenia Bulletin, [S.1.], v. 6, n. 3, p. 490-505, 1980.

BANDURA, A. Social learning theory. New Jersey: Prentice-Hall, 1977.

BARROWCLOUGH et al. Assessing relatives' needs for psychosocial interventions in schizophrenia: a relatives' version of the Cardinal Needs Schedule (RCNS). Psychol Med, v. 28, n. 3, p. 531-542, May. 1998.

BARROWCLOUGH et al. Randomised controlled effectiveness trial of a needsbased psychosocial intervention service for carers of people with schizophrenia. British Journal of Psychiatry, [S.1.], v. 174, p. 505-511, 1999.

BARROWCLOUGH, C; TARRIER, N. Families of schizophrenic patients: cognitive behavioural intervention. London: Nelson Thornes, 1997.

BECK, A.T. Cognitive therapy and the emotional disorders. Boston: Internat. Universities Press, 1979.

BROWN, G.W.; BIRLEY, J.L.T.; WING, J.K. Influence of family life on the course of schizophrenic disorders: A Replication. British Journal of Psychiatry, [S.1.], v. 121, p. 241-258, 1972.

BUTZLAFF, R.L.; HOOLEY, J.M. Expressed emotion and psychiatric relapse: a meta-analysis. Arch Gen Psychiatry, [S.1.], v. 55, n.6, p. 547/552, Jun. 1998.

CABALLO, V. E. (Org.). O treinamento em habilidades sociais. In: . Manual de técnicas de terapia e modificação do comportamento. São Paulo: Santos, 1996. p. 361-398. 
CHIEN, W.; CHAN, S.W.C. One-year follow-up of a multiple-family-group intervention for chinese families of patients with schizophrenia. Psychiatric Service, Arlington, v. 55, n. 11, p. 1276-1284, 2004.

DIXON, L. B. Providing services to families with schizophrenia: present and future. J Mental Health policy Econ, [S.1.], v. 2, p. 3-8, 1999.

DIXON, L.B.; LEHMAN, A.F. Family interventions for schizophrenia. Schizophrenia Bulletin, [S.1.], v. 21, p. 4; 631-643, 1995.

DOBSON, K.S.; DOZOIS, D.J. Historical and philosophical bases of the cognitivebehavioral therapies. In.: DOBSON, K.S. (Ed.). Handbook of cognitive-behavioral therapies. New York: The Guilford Press, 2001. p. 3-39.

FALLOON, I.R.H.; PEDERSON J. Family management in the prevention of morbidity of schizophrenia: the adjustment of the family unit. British Journal of Psychiatry, [S.1.], v.147, p.156-163, 1985.

FALLOON, I.R.H. et al. Family management in the prevention of morbidity of schizophrenia: clinical outcome of a two-year longitudinal study. Archives of General Psychiatry, [S.1.], v. 42, n. 9, p. 887-896, 1985.

. Family management in the prevention of exacerbations of schizophrenia: a controlled study. The New England Journal of Medicine, [S.1.], v. 306, n. 24, p. 1437-1440, 1982.

GLICK, I.D. et al. A controlled evaluation of inpatient family intervention. Arch Gen Psychiatry, [S.1.], v. 42, p. 882-887, 1985.

GOLDSTEIN, M.J. et al. Drug and family therapy in the aftercare of acute schizophrenia. Arch Gen Psychiatry, [S.1.], v. 35, p. 1169-1177, 1978.

HAAS, G.L. et al. Inpatient family intervention: a randomized clinical trial. Arch Gen Psychiatry, [S.1.], v. 45, p. 217-224, 1988.

HOGARTY, G.E. et al. Personal indicators in the course of Schizophrenia Research Group: family psychoeducation, social skills training and maintenance chemotherapy in the aftercare treatment of schizophrenia. I. One-year effects of a controlled study on relapse and expressed emotion. Archives of General Psychiatry, [S.1.], v. 43, n. 7, p. 633-642, 1986.

. Family psychoeducation, social skills training, and maintenance chemotherapy in the aftercare treatment of schizophrenia. Archives of General Psychiatry, [S.1.], v. 48, n. 4, p. 340-347, 1991.

KRAUSS-SILVA, L. Avaliação tecnológica em saúde: questões metodológicas e operacionais. Cad. Saúde Pública, Rio de Janeiro, v. 20, supl. 2, p. 199-207, 2004.

LEFF, J. et al. A trial of family therapy versus a relatives' group for schizophrenia. Two-year follow-up. Br J Psychiatry, [S.1.], v.157, p. 571-577, Oct. 1990. 
LEFF, J. et al. A trial of family therapy v. a relatives group for schizophrenia. Br J Psychiatry, [S.1.], v. 154, p. 58-66, Jan. 1989.

. A controlled trial of social intervention in the families of schizophrenic patients: two-year follow-up. British Journal of Psychiatry, [S.1.], v. 146, p. 594600, 1985.

. A controlled trial of social interventions in the families of schizophrenic patients. British Journal of Psychiatry, [S.1.], v. 141, p. 121-134, 1982.

LEHMAN, A.F. et al. At issue: translating research into practice: the schizophrenia patients outcome research team (PORT). Treatment Recommendations. Schizophrenia Bulletin, [S.1.], v. 24, n.1, p. 1-10, 1998.

MCFARLANE, W.R. et al. Multiple-family groups and psychoeducation in the treatment of schizophrenia. Arch Gen Psychiatry, [S.1.], v. 52, p. 679-687, 1995.

MERINDER, L.B. et al. Patient and relative education in community psychiatry: a randomized controlled trial regarding its effectiveness. Soc Psychiatr Epidemiol, [S.1.], v. 34, p. 287-294, 1999.

MÖNKING, H.S. Self-help groups for families f schizophrenic patients: formation, development and therapeutic impact. Soc Psychiatr Epidemiol, [S.1.], v. 29, p. 149-154, 1994.

MONTERO, I. et al. Do all people with schizophrenia receive the same benefit from different family intervention programs? Psychiatry Res., [S.1.], n. 28, v. 133, n. 2-3, p. 187-195, Feb. 2005.

. Two strategies for family intervention in schizophrenia: a randomized trial in a Mediterranean environment. Schizophr Bull, [S.1.], v. 27, n.4, p. 661-670, 2001.

MUESER, K.T. et al. Family treatment and medication dosage reduction in schizophrenia: effects on patient social functioning, family attitudes, and burden. Journal of Consulting and Clinical Psychology, Washington, v. 69, n.1, p. 3-12, 2001.

RAN, M. et al. Effectiveness of psychoeducational intervention for rural chinese families experiencing schizophrenia: a randomized controlled trial. Soc Psychiatr Epidemiol, [S.1.], v. 38, p. 68-75, 2003.

SCHENE, A.H., WIJNGAARDEN, B., KOETER, M.W.J. Family caregiving in Schizophrenia: domains and distress. Schizophrenia Bulletin, [S.1.], v. 24, n. 4, 1998.

SCHENE, A.H., TESSLER R.C., GAMACHE G.M. Instruments measuring family or caregiver burden in severe mental illness. Soc Psychiatry Psychiatr Epidemiol, [S.1.], v. 29, p. 228-240, 1994. 
SHIMODERA, S. et al. Expressed emotion and psychoeducation intervention for relatives of patients with schizophrenia: a randomized controlled study in Japan. Psychiatry Research, [S.1.], v. 96, p. 141-148, 2000.

SELLWOOD, W. et al. Needs-based cognitive-behavioral family intervention for carers of patients suffering from schizophrenia: 12-month follow-up. Acta Psychiatr Scand, [S.1.], v. 104, p. 346-355, 2001.

SZMUKLER, G.I. et al. A controlled trial of a conselling intervention for caregivers of relatives with schizophrenia. Soc Psychiatr Epidemiol, [S.1.], v. 31, p. 149-155, 1996.

TARRIER, N. et al. The community management of schizophrenia: a controlled trial of a behavioural intervention with families to reduce relapse. British Journal of Psychiatry, [S.1.], v. 153, p.532-542, 1988.

TOMARAS, V. et al. The effect of family intervention on chronic schizophrenics under individual psychosocial treatment: a 3-year study. Soc.Psychiatry and Psychiatr. Epidemiology, [S.1.], v. 35, p. 487-493, 2000.

U. S. DEPARTMENT OF HEALTH AND HUMAN SERVICES. Mental health: a report of the surgeon general - executive summary. Rockville, MD: U.S. National Institute of Mental Health, 1999.

VAUGHAN, K. et al. The Sydney intervention trial: a controlled trial of relatives' counselling to reduce schizophrenic relapse. Soc Psychiatry Psychiatr Epidemiol., [S.1.], v. 27, n. 1, p. 16-21, Jan. 1992.

VAUGHN, C.; LEFF, J. The measurement of expressed emotion in the families of psychiatric patients. British Journal of Social and Clin Psychology, [S.1.], v. 15, p. 423-429, 1976.

XIONG, W. et al. Family-based intervention for schizophrenic patients in China: a randomised controlled trial. British Journal of Psychiatry, [S.1.], v. 165, p. 239247, 1994.

ZHANG, M. et al. Randomized-control trial of family intervention for 78 FirstEpisode male schizophrenic patients. British Journal of Psychiatry, [S.1.], v. 165, suppl. 24, p. 96-102, 1994.

Recebido em: março/ 2005.

Aceito em: junho/ 2006. 
